\title{
Reflets
}

Revue d'intervention sociale et communautaire

\section{De peine et de misère : structures sociales et souffrance au travail dans le capitalisme contemporain}

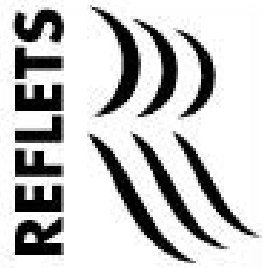

\section{François Boudreau}

Volume 19, numéro 2, automne 2013

La souffrance psychique et morale au travail : enjeux pour les professionnels du secteur de la santé et des services sociaux

URI : https://id.erudit.org/iderudit/1021179ar

DOI : https://doi.org/10.7202/1021179ar

Aller au sommaire du numéro

Éditeur(s)

Reflets, Revue d'intervention sociale et communautaire

ISSN

1203-4576 (imprimé)

1712-8498 (numérique)

Découvrir la revue

Citer cet article

Boudreau, F. (2013). De peine et de misère : structures sociales et souffrance au travail dans le capitalisme contemporain. Reflets, 19(2), 44-71.

https://doi.org/10.7202/1021179ar

\section{Résumé de l'article}

Il est possible d'étudier la souffrance au travail dans le but de remédier à une forme spécifique de souffrance dans le cadre d'un travail particulier. Mais si c'est la catégorie de travail qui est synonyme de souffrance pour la majorité de la population dans une société donnée, si c'est le sens de cette activité qui se soustrait à l'entendement, c'est qu'il y a un problème avec la nature du travail, avec sa conception et avec l'organisation du travail lui-même. Se soustraire à la souffrance au travail passe alors par l'évaluation du sens de l'activité travail « pour la société ».
Tous droits réservés (C) Reflets, Revue d'intervention sociale et communautaire, 2013 cest protégé par la loi sur le droit d'auteur. L'utilisation des services d'Érudit (y compris la reproduction) est assujettie à sa politique d'utilisation que vous pouvez consulter en ligne. 


\title{
De peine et de misère : structures sociales et souffrance au travail dans le capitalisme contemporain
}

\author{
François Boudreau \\ Sociologue/Professeur agrégé \\ École de service social, Université Laurentienne
}

L'utopie industrialiste nous promettait que le développement des forces productives et l'expansion de la sphère économique allaient libérer l'humanité de la rareté, de l'injustice et du mal-être; [...] De cette utopie il ne reste rien. (Gorz, 1988, p. 25)

\section{Résumé}

Il est possible d'étudier la souffrance au travail dans le but de remédier à une forme spécifique de souffrance dans le cadre d'un travail particulier. Mais si c'est la catégorie de travail qui est synonyme de souffrance pour la majorité de la population dans une société donnée, si c'est le sens de cette activité qui se soustrait à l'entendement, c'est qu'il y a un problème avec la nature du travail, avec sa conception et avec l'organisation du travail lui-même. Se soustraire à la souffrance au travail passe alors par l'évaluation du sens de l'activité travail "pour la société ".

Mots clés : Souffrance, travail, capitalisme, aliénation, endettement, service social.

\section{Abstract}

It is possible to study the suffering at work with the aim of addressing a specific form of suffering in the context of a particular 
job. But if the job as a category of activity that is synonymous with suffering for the majority of the population in a given society, if it is the meaning of this activity that disappear from human understanding, it is that there is a problem with the nature of work, with its purpose and with the organization of the work itself. Therefore, the cure to the suffering at work passes by a profound reassessment of the meaning of work as an activity "for society".

Key words : Suffering, work, capitalism, alienation, debt, social work.

\section{Épistémologie : deux manières de réfléchir la souffrance au travail}

L'étude de la souffrance au travail peut procéder d'au moins deux manières. Il est possible d'étudier la souffrance au travail « en soi ", c'est-à-dire comme phénomène isolé et particulier qui qualifie une dimension pathologique d'un tel travail, tel que ce travail est aujourd'hui organisé et structuré dans la société. Une telle étude pourrait par exemple chercher à saisir leslimites à l'intérieur desquelles il demeurerait souhaitable de maintenir le «travail », de manière à circonscrire, à corriger et à atténuer ses lacunes afin de le maintenir comme une importante activité humaine génératrice de sens. Ce genre d'étude viserait à corriger la pathologie inhérente à une forme de travail de nature exceptionnelle. C'est généralement ce que font par exemple les études sur la conciliation travail/ famille, ou encore celles visant une réduction de l'absentéisme au travail.

L'étude de la souffrance au travail pourrait procéder autrement, ou plutôt, on pourrait y ajouter une autre dimension en effectuant une analyse "pour-so ${ }^{1}$ » du travail, c'est-à-dire avec l'objectif de comprendre non pas la pathologie liée à tel ou à tel travail ou la pathologie de certains secteurs de travail, mais le sens de l'activité humaine qualifiée de "travail " dans la vie en général et dans la société concrète. Pour cela, il faut ouvrir la réflexion de manière beaucoup plus large et voir à quelles conditions historiques 
«Nous abordons la question de la souffrance psychique au travail en tant qu'indicateur d'une perte de sens pour l'individu qui l'accomplit..." "Nous dirons que la souffrance psychique généralisée au travail découle de l'organisation et de la finalité du travail contemporain et que ce n'est donc pas telle ou telle dimension du travail qui est mal organisée (pathologique)...» générales correspond cette forme de souffrance. Par exemple, en se posant la question de savoir si la souffrance psychique si largement répandue aujourd'hui a remplacé la souffrance physique à partir du moment où le capitalisme financier et la nouvelle gestion se sont superposés au capitalisme industriel et au taylorisme au tournant des années 1980.

Contre les tendances positivistes et sociométriques, le présent article propose une réflexion pour-soi et subjective, donc à visée normative, de la souffrance psychique au travail, qui vise à mettre en évidence un parti pris épistémologique de la réflexion théorique en sciences sociales. Nous abordons la question de la souffrance psychique au travail en tant qu'indicateur d'une perte de sens pour l'individu qui l'accomplit dans un milieu et dans des conditions données, ainsi que sous l'angle d'une perte de sens pour une société qui adopte une forme spécifique de division du travail. Nous considérons que ce qui doit être mis en évidence, au-delà des manifestations particulières et empiriques de la souffrance au travail, de tel ou tel travail, c'est le sens - de fait, sa perte de sens - de l'activité travail dans la société contemporaine. Nous dirons que la souffrance psychique généralisée au travail découle de l'organisation et de la finalité du travail contemporain et que ce n'est donc pas telle ou telle dimension du travail qui est mal organisée (pathologique), mais l'organisation même du travail et ses finalités pour la société qui sont déphasées au regard de la nature humaine anthropologique.

Pour bien mettre tout cela en évidence, il nous faudra comprendre les transformations de sens que l'activité travail a subies au fil des transformations anthropologiques et historiques de la société, auxquelles correspondent des transformations de ses modes d'être. Il apparaitra alors que nous sommes vraisemblablement parvenus à épuiser les formes de sens que peut porter le type de travail radicalement divisé et parcellarisé sur lequel la société contemporaine fonde sa légitimité et qui caractérise le rapport contemporain de l'être au travail.

De manière plus concrète, après avoir mesuré la place et le rôle du travail dans sa dimension anthropologique et historique, nous évaluerons la stratégie adoptée par la société capitaliste 
pour acquérir sa légitimité, stratégie qui consiste à répondre aux besoins illimités que rencontre l'Homme ${ }^{2}$ en société (Rosanvallon, 1981; Gorz, 1988). Nommément, le capitalisme s'est instauré comme système capable de "livrer la marchandise ". Mais ainsi, pour paraphraser Freitag (2007), le "progrès » et sa connotation normative véhiculée par la société moderne depuis le $\mathrm{XVI}^{\mathrm{e}}$ siècle ont cédé la place au "développement " et à sa connotation empirique dans la société contemporaine depuis la fin du XIX siècle. La finalité contenue dans le progrès, compris comme ce qui devrait être "qualitativement " différent du statu quo, a de même cédé sa place au processus du développement, compris comme "quantitativement " supérieur à ce qu'il y avait. Nous sommes passés du "toujours mieux " au "toujours plus ».

L'analyse de ce changement ou de ce passage nous aidera à comprendre l'enracinement présent de la souffrance au travail et cela, de deux manières. En saisissant d'abord que la société de consommation implique une société de surproduction, provoquant d'évidentes conséquences négatives sur les êtres humains, qui doivent maintenir la cadence infernale de la production des choses, des services ou du savoir et de leur consommation (Rodin, 2011).

"...la pathologie réside largement dans la préférence accordée à une conception quantitative ou matérielle de la vie, plutôt que dans une conception qualitative ou normative se référant à des valeurs spirituelles ou métaphysiques. " En comprenant ensuite que la pathologie réside largement dans la préférence accordée à une conception quantitative ou matérielle de la vie, plutôt que dans une conception qualitative ou normative se référant à des valeurs spirituelles ou métaphysiques. Nous voulons ici faire écho à Weber (1904) et à ses catégories d'action par rapport à une finalité et à des valeurs ${ }^{3}$ pour souligner que c'est vraisemblablement là que réside une partie de notre réponse. En effet, la dimension quantitative de la vie, «la nécessité impérative de son augmentation continue par la croissance économique » diraient les apôtres du développement capitaliste, impose une accélération ou une intensification insoutenable de la vie. Il y a dans cette conception beaucoup de souffrance psychique en acte et en réserve.

Revenons un l'instant à l'observation de Freitag sur la transformation récente des paramètres de la vie collective, car elle contient une importante piste de réflexion quant à la difficulté rencontrée par les sciences sociales à appréhender réellement leur objet d'étude d'une manière qui ne les mène pas directement à 
"Les spécialistes des sciences sociales n'ont pas vocation à devenir des médecins du système, chargés de la normalisation des excentrés, des marginalisés et des victimes de ce système. " devenir, elles aussi, agentes du système. Le même glissement qui a vu la société moderne passer de préoccupations d'ordre qualitatif à des préoccupations d'ordre quantitatif a affecté les sciences sociales lorsque la compréhension devant évaluer et légitimer la normativité moderne a laissé la place à la mesure qui quantifie les accomplissements du système. Le danger qui guette alors les sciences sociales, tel que se caractérise massivement sa tendance dominante depuis un demi-siècle, est de cautionner un système qui transforme les chercheurs en spécialistes de la gestion des rapports sociaux. Les sciences sociales deviennent ainsi un outil supplémentaire de la nouvelle gestion qui facilite la participation des "opprimés " dans la quête d'un travail moins pénible. Les spécialistes des sciences sociales n'ont pas vocation à devenir des médecins du système, chargés de la normalisation des excentrés, des marginalisés et des victimes de ce système.

La sociologie, voire l'ensemble des sciences sociales, a généralement suivi les orientations du capitalisme au $\mathrm{XX}^{\mathrm{e}}$ siècle en cédant sa finalité, qui est l'évaluation de l'orientation normative (du sens), à un processus toujours plus approfondi d'intégration dans la gestion du système (Caillé, 1986; Freitag, 2007), phénomène qu'avait largement vu se développer Gouldner dès 1970. La sociologie, comme l'ensemble des sciences sociales, en particulier à partir de la pression universitaire pour la recherche (Freitag, 1995; Gagné, 1999; Caillé et Chanial, 2009), travaille aujourd'hui trop souvent à l'intégration des individus, des groupes ou des collectivités qui ont été excentrés du monde. La sociologie doit se réapproprier son objet, c'est-à-dire l'évaluation de la normativité de la "totalité » de la société, et ne plus se limiter à l'étude de milieux sociaux spécifiques où s'expriment les pratiques particulières, entièrement empiriques et quantifiables.

\section{Méthodologie : lier l'objectivité et la subjectivité de l'étude dans la recherche du sens}

L'étude de la souffrance au travail ne doit pas seulement être abordée "objectivement " dans ses formes, ses manifestations 
ou sa fréquence. Elle doit en plus être saisie "subjectivement", afin de comprendre son sens pour l'individu et la société, dans leur développement historique et dans leurs finalités. Évoquons à nouveau Weber pour méditer ses propos qui ramènent à nouveau la profession de "savant du social » à des considérations ontologiques $^{4}$, notamment au sujet du sens de la mort :

Les paysans d'autrefois sont morts "vieux et comblés par la vie " parce qu'ils étaient installés dans le cycle organique de la vie, parce que celle-ci leur avait apporté au déclin de leurs jours tout le sens qu'elle pouvait leur offrir et parce qu'il ne subsistait aucune énigme qu'ils auraient encore voulu résoudre. Ils pouvaient donc se dire satisfaits de la vie. L'Homme civilisé au contraire, placé dans le mouvement d'une civilisation qui s'enrichit continuellement de pensées, de savoirs et de problèmes, peut se sentir «las » de la vie et non pas " comblé " par elle. En effet, il ne peut jamais saisir qu'une infime partie de tout ce que la vie de l'esprit produit sans cesse de nouveau, il ne peut saisir que du provisoire et jamais du définitif. C'est pourquoi la mort est à ses yeux un évènement qui n'a pas de sens. (Weber, 1917, p. 71)

Ainsi, nous revenons au sens de l'existence qui s'impose à chacun et qui est inexorablement lié à la société dans laquelle chacun de nous "vient au monde ». Sans une existence sensée, pourrions-nous dire, il ne peut y avoir d'existence heureuse. En l'absence de sérénité, il n'y a que malaise, inconfort, douleur, voire souffrance. Reposons donc la question : quel est le sens du travail aujourd'hui? Quelle est sa place dans la vie de chacun? Nous sommes nombreux à entendre le commentaire, venant de nos collègues, des membres de notre famille, de nos voisins, voire des médias, selon lesquels "ça n’a plus de sens ", le «ça " désignant parfois la vie et le monde, parfois les décisions des gestionnaires qui visent le perfectionnement de la productivité. Nous sommes 
«Nous sommes nombreux à entendre le commentaire, venant de nos collègues, des membres de notre famille, de nos voisins, voire des médias, selon lesquels "ça n'a plus de sens ", le "ça» désignant parfois la vie et le monde..."

\section{"La finalité de}

l'Homme dans la société archaïque, telle que racontée dans les mythes ${ }^{5}$, consiste à ne pas déranger l'ordre du monde ou à remettre le monde en ordre par divers rituels. " nombreux à entendre d'autres variantes de cette expression, du genre : «je n'en peux plus! ». Serait-ce que le travail a perdu son sens pour l'individu, tant et si bien que ce dernier ne comprend plus pourquoi ou pour qui il travaille tant? Serait-ce que dans ces conditions, l'individu souffre de ne plus comprendre le sens de ses actions, de ses attaches au travail et de son appartenance au monde? Serait-ce que, paradoxalement, l'individu se lasse de courir pour gagner sa vie et pour dépenser le fruit de son labeur dans la consommation des marchandises?

Le mythe de la société archaïque, la religion de la société traditionnelle et la science de la société moderne ont quelque chose d'extraordinaire en commun : chacun raconte à sa manière sa société, sa propre histoire totale, de sa genèse à la fin des temps, et chacun est convaincu de détenir " toute la vérité "sur son monde. Par exemple, dans le mythe de la société archaïque, la genèse est généralement fantasmée comme résultat de l'action d'un démiurge : un être qui tombe des nuages (Aataentsic chez les Wendat); un arbre qui pousse et qui donne la diversité de la vie (Yggdrasil des cultures nordiques); ou l'eau salée qui rencontre l'eau douce, donnant ainsi naissance à tous les êtres (Mésopotamie). La finalité de l'Homme dans la société archaïque, telle que racontée dans les mythes ${ }^{5}$, consiste à ne pas déranger l'ordre du monde ou à remettre le monde en ordre par divers rituels (Éliade, 1963; Hallpike, 1979). Dans les diverses religions des sociétés traditionnelles, c'est un dieu qui est le personnage central. Pour l'Égypte ancienne, Ré, le démiurge ou créateur incréé, fait sortir de lui-même le premier couple de dieux, Shou et Tefnout, grâce à son sperme obtenu par masturbation (il créa le plaisir). Une autre version du mythe affirme que ces premières divinités sont nées du crachat de Ré. Quant aux Hommes, ils seraient nés de ses larmes. Dans le cas du judaïsme et des deux autres religions abrahamiques (christianisme et islam), Dieu crée le monde en six jours, et l'Homme vient à l'existence au sixième jour. Ces religions postulent une fin du monde consacrée par un jugement dernier qui consistera à séparer ceux qui auront respecté les commandements et ceux qui en auront été incapables. Dans 
ce contexte, la finalité de l'Homme est de suivre des injonctions venues d'ailleurs.

Dans la société rationnelle moderne, la présence humaine sur Terre se fonde sur une explication scientifique et universelle : l'origine débute avec le Big Bang et la vie découle d'un long processus naturel, l'humain et les autres animaux étant le résultat d'une évolution fondée sur la sélection naturelle et sur les autres contingences de la nature. Historiquement, il aura fallu attendre le développement des sciences sociales pour que les finalités de l'Homme puissent être synthétisées à partir de ce qui a toujours été valorisé dans la culture humaine : vivre en paix, en harmonie, en solidarité et en respect. Ce que les différentes chartes des droits et les différentes lois sociales ont maintes fois tenté de mettre en forme.

Comme on le voit, chacune de ces modalités discursives porte sur la totalité de la société, du point de vue de cette même société. Chaque modalité discursive de chacun des types de société rend compte du sens profond que se donne à lui même chacun de ces types de société, pour ceux qui vivent dans cette société. On peut aussi élaborer une typologie de la conception de la famille propre à chacun de ces types de société, une typologie de leurs conceptions du mariage, de leurs rites de naissance, de puberté, de funérailles et de bien d'autres choses encore. Tout cela permet de dégager un contexte général dans lequel se saisit ce qui distingue les types de sociétés. On peut également, comme nous le ferons maintenant, y dévoiler le sens que chacun d'eux donne au travail, aux transformations fondamentales de son contenu dans le temps et à sa place pour l'individu dans son rapport au monde.

Cela nous permet non seulement de voir le (non) sens de la situation actuelle, mais aussi de comprendre les limites de la "scientificité des études sur le travail " lorsque ce dernier est considéré en soi. La réflexion doit nous porter vers un retour sur le sens : il nous faut revenir à la finalité des sciences sociales, qui consiste à évaluer normativement la société. Il est alors possible de formuler la question centrale à laquelle il importe de répondre : le travail tel qu'il est organisé aujourd'hui conserve-t-il un sens du point de vue de l'humanitude, c'est-à-dire, du point de vue de ce 
que signifie être humain? La soi-disant évolution consiste-t-elle simplement à perfectionner l'organisation du travail humain pour la production? Le travail devrait-il permettre à l'individu de se réaliser "soi-même ", ou au contraire, s'inscrit-il dans la logique de la productivité "pour l'autre " qui n'a plus rien à voir avec la dimension anthropologique de cette activité nommée travail? Cette réflexion nous pousse également à réfléchir à nouveau le sens et l'usage des sciences sociales. Continueront-elles à poser la question de la normativité ou serviront-elles à une meilleure intégration de l'individu dans un système qui le dépasse?

\section{Le travail : perspective anthropologique et historique}

"Le temps de travail moyen de certaines communautés archaïques était de l'ordre de 3 à 4 heures par jour, et ce travail était généralement effectué en commun et pour tous. »
Nous pensons généralement la communauté archaïque comme une société du manque, de la pauvreté, de la misère. Or, c'est là une fausse conception. L'économie des sociétés primitives est adaptée aux besoins immédiats des hommes et des femmes. Le groupe, fondé sur le système familial, organise la solidarité. Les ressources disponibles le sont pour tous. De manière générale, nous pouvons donc dire qu'à l'origine, l'Homme est l'objectif de la production alors qu'aujourd'hui c'est l'inverse : l'Homme est devenu un moyen de la production (entre autres, Sahlins, 1976).

Le temps de travail moyen de certaines communautés archaïques était de l'ordre de 3 à 4 heures par jour, et ce travail était généralement effectué en commun et pour tous (Clastres, 1974). Certains Autochtones masculins du Brésil travaillaient (défricher un lopin de terre à la hache de pierre et par le feu) pendant l'équivalent d'un à deux mois tous les quatre à six ans. Les descriptions de Trigger (1991) du travail des communautés huronnes sont en tous points similaires à celles de Clastres (1974). Si les Hommes "peinent au travail ", qui est défini comme défrichage de la forêt et cueillette, il est bien difficile de définir avec quelques précisions une activité régulière et quotidienne que nous nommerions travail au sens contemporain du terme. Tout 
comme pour les hommes aujourd'hui, la chasse et la pêche ne peuvent être décrites ainsi ${ }^{6}$, pas plus que ne le sont les activités artisanales, la fabrication des ornements ou des outils par les femmes. Ces activités ritualisées ne sont pas des peines (ponos), mais relèvent d'un échange avec le monde. Elles sont créatives, effectuées en groupe, dans la collégialité et en l'absence de hiérarchie et de contrainte.

D'une certaine manière encore, le don de la communauté archaïque participe totalement de cette conception unitaire, collégiale et circulaire du travail ou de l'activité productive (Mauss, 1960). Si le chasseur ruse d'habileté avec l'orignal pour être en mesure de le coincer, et que sa flèche, plus rapide, déjoue l'anticipation de l'animal, c'est qu'au-delà de son habileté, il profite des grâces des esprits. La capture de l'animal n'est alors pas le résultat de la seule activité du chasseur, mais aussi de la générosité des puissances en acte :le vent favorable, l'esprit de la flèche ou la volonté de l'animal. Le partage des prises de la chasse devient alors une reconnaissance de la générosité des puissances qui participent au succès du chasseur. C'est aussi la meilleure façon de s'assurer que ces puissances demeurent favorables aux chasseurs. Voilà le genre de conceptions totalement niées par l'activité «travail » dans le monde moderne (Godbout et Caillé, 1992).

L'expérience des Grecs viendra complexifier considérablement la compréhension du rapport de l'Homme à la nature. Chez les Grecs, on peut distinguer au moins trois niveaux de la réalité " économique " qui ne sont pas encore "travail ", mais plutôt des activités particulières, chacune avec son sens et ses modalités (Freitag, 1989). D'abord, il y a la conception de l'ergazasthai, directement et exclusivement associée au travail arboricole, activité à double dimension externe à la chose «en soi ». Dans ce type d'activité, l'activité humain assiste d'abord matériellement la manifestation autonome d'une puissance en acte dans l'arbre fruitier, il n'est pas la source de cette manifestation. Son rôle dans l'assistance de cette puissance se traduit par différentes activités de type instrumental, tels l'engraissement et l'émondage.

Par une attitude d'accueil respectueuse (de don et de contredon), l'Homme participe ensuite rituellement à cette 
« L'Homme approfondit ainsi sa communion avec les dieux et sa participation est généralement festive, effectuée dans le respect $d u$ "faire correctement ».» manifestation de la puissance en acte. Cette double dimension de l'activité, matérielle et rituelle, facilite l'activité autonome de la puissance qui génère (genos) elle-même sa finalité dans sa manifestation particulière, dans le fait de produire des fruits par exemple. L'Homme approfondit ainsi sa communion avec les dieux et sa participation est généralement festive, effectuée dans le respect du " faire correctement ». Cette conception immédiatement spirituelle découle du fait que l'activité n'est pas directement productive; l'arbre pousse seul et c'est lui, et non l'Homme, qui produit les fruits. C'est ainsi que la conception n'est pas celle du labeur, mais de la participation de l'individu au processus général, à la manière du don de nature et du contredon de l'Homme.

Ensuite, il y a le ponos, la peine, qui est directement associé à l'activité agricole où l'effort à déployer est pénible, mais aussi distinct de la jouissance des fruits du labeur. Cette conception est en relation avec la déesse Demeter (Harrison, 1991) qui garantit la moralité de celui qui travaille la terre en faisant de sa peine un pont avec le divin.

Ainsi, dans la Grèce antique, l'activité au regard des produits de la terre fait apparaitre deux formes d'expérience. Le premier pôle de déploiement de cette expérience, l'ergazasthai, prend la forme d'une communication de l'Homme avec la nature, qui fait alors « apparaitre les choses » comme issues de puissances créatrices autonomes de la nature. Cette expérience est vécue comme une manifestation de la puissance en acte, comme prolongement de l'activité de cueillette qui est don de la nature. Le second pôle de déploiement de cette expérience à la terre, le ponos, est l'agriculture, le travail de la terre dans lequel la peine s'oppose à la praxis, à la guerre et à la palabre, qui sont des activités relevant de la liberté issue directement de l'Homme (Vernant, 1965; Freitag, 1989).

Un troisième type d'activité que les Grecs distinguent est celui de la production artisanale, la poesis, impliquant dans sa conception une autonomie de l'activité humaine qui devient un "faire ", ou un «fabriquer» auquel correspond la volonté de la cause efficiente. 
"Lorsque nous évoquons la souffrance au travail, il s'agit d'une variante $d u$ ponos, de cet effort du corps et de l'esprit qui sépare l'activité de son résultat " pour un autre »."

"...le travail d'alors permettait de vivre: les individus travaillaient pour vivre. Que va-t-il donc se passer pour que nous en arrivions à la situation actuelle où nous vivons pour travailler?"
Lorsque nous évoquons la souffrance au travail, il s'agit d'une variante du ponos, de cet effort du corps et de l'esprit qui sépare l'activité de son résultat "pour un autre ». L'effort, la «peine » et le «labeur» des activités productives relevant de l'ergazasthai, d'un rapport de don et de contredon avec la nature ou avec l'autre, ne dissocie pas le «faire ", l'« avoir » et l'« être » de l'individu qui peine. Ce type d'activité ne divise pas l'individu pour le faire exister dans des moments détachés de la vie. Il ne le rend pas dépendant des autres pour être complètement, totalement Homme. La preuve en est que nous retournons joyeusement jardiner à la maison pour faire disparaitre nos symptômes d'aliénation après une journée au travail.

Dans la société traditionnelle, sous l'influence de la doctrine chrétienne, «le travail est la punition que Dieu inflige à l'Homme en le chassant du paradis » (Jacob, 1994) et c'est toujours l'effort au travail, la peine plutôt que le résultat, qui est valorisé. Bien que marqué par d'importantes variations selon les années et les lieux précis pour l'Europe, selon les aléas climatiques et les formes précises des rapports sociaux, le temps de travail dépasse alors tout juste un jour sur trois, avec une médiane de 125,8 jours dans l'année (Sahlins, 1976). Le travail en vient progressivement à désigner une activité utile, en remplacement d'une activité pénible à laquelle il connotait avant, et le concept prend aussi un sens nettement utilitariste dès la fin du Moyen Âge (Jacob, 1994). Mais même si ce travail demeure ponos, un travail pour l'autre, un constat s'impose tout de même ici, celui d'un renversement annoncé; le travail d'alors permettait de vivre : les individus travaillaient pour vivre. Que va-t-il donc se passer pour que nous en arrivions à la situation actuelle où nous vivons pour travailler? Comment expliquer cette inversion de sens?

\section{Le libre arbitre et le renversement de l'activité sociale dans la modernité}

Lorsque la modernité s'installe à partir du XVI siècle, c'est précisément contre la tradition, contre son sens et contre son 
"Mais lorsque

l'activité est libre, fondée sur le sens que lui donne l'individu, il n'y a plus de sens collectif possible, le contenu d'une activité est alors indéterminé... » arbitraire, contre ses pratiques aussi, contre ses formes de contrôle social, bref, contre son ordre et ses normes. Mais dire que les anciennes normes étaient fondées dans la tradition, c'est aussi dire qu'elles relevaient d'une création " collective ", que leur mise en place avait été lente, cumulative, arbitraire certes, mais " enracinée " dans l'histoire. Or, le fondement de la normativité moderne ne sera plus déposé dans la culture du groupe, où chaque génération reproduit le geste ancestral dont la conformité se pose devant toutes les générations qui le précèdent. La normativité ne sera plus déposée dans le cœur de Dieu, dans la nécessité de se racheter du péché originel, là où tous les Hommes demeurent unis dans leur socialité terrestre autant que dans le paradis. La nouvelle normativité sera déposée entre les mains, ou plutôt dans la conscience, d'individus libres ${ }^{7}$. Cette situation radicalement nouvelle consiste à permettre à chacun de poursuivre sa «libre volonté ». C'est la première fois dans toute l'histoire de l'humanité que l'Homme est en droit de poursuivre sa destinée, ses activités, comme il lui convient.

Cette situation est cependant problématique, parce que cette libre volonté n'aura comme seul compas moral que le "libre arbitre » de chaque individu et la totale autonomie de son exercice face à toutes les normes concrètes de la société et à toutes les contingences de la vie. Lorsque l'appartenance de chacun au groupe posait le travail comme modalité de contribution à l'existence de tous, cette activité avait un sens concret et précis pour chacun en tant que membre du tout. Lorsque la croyance en Dieu posait le travail comme modalité du rachat du péché, l'activité maintenait ce sens collectif; tous étaient soumis à cette contingence, tous y trouvaient une identité commune, une appartenance commune, un sens commun. Mais lorsque l'activité est libre, fondée sur le sens que lui donne l'individu, il n'y a plus de sens collectif possible, le contenu d'une activité est alors indéterminé; "par définition elle ne peut trouver un contenu qu'en dehors d'elle-même, dans une "nécessité objective", une "pure positivité", et ce contenu ne peut alors en tant que tel valoir a priori, normativemen » (Freitag, 1987, p. 20). Conséquemment, ce libre arbitre fera en sorte que chacun cherche son bonheur pour 
lui-même, sans se préoccuper de la conscience collective et du sens d'appartenance à une seule et même entité. Ne manque alors qu'une justification philosophique articulée qui fera la promotion de l'individualisme pour que toute la société soit entrainée dans cette dérive.

C'est Locke (1690, \ 28) qui donnera l'impulsion libérale essentielle du travail comme activité isolée de la société, en lui attribuant le déterminant de la propriété : «Le travail, qui est mien, mettant ces choses hors de l'état du commun, les a fixées et me les a appropriées.» Par le travail,l'Homme « rend ce bien-là son bien particulier, et le distingue de ce qui est commun à tous " ( $\mathbb{S} 32$ ). Locke pose la relation à établir entre le travail et la propriété en affirmant que le droit à la propriété est inscrit en l'Homme : " l'Homme néanmoins, étant le maitre et le propriétaire de sa propre personne, de toutes ses actions, de tout son travail, a toujours en soi le grand fondement de la propriété " (\$ 44$)$.

Cette conception de l'activité humaine dans le monde, du rapport entre travail, richesse et propriété, constitue une large synthèse de l'activité marchande telle que cette activité se déploie alors en Europe. De là, la trame historique est mieux connue : l'Europe préside au développement de l'activité capitaliste qui transforme de plus en plus la vie elle-même en rapport marchand organisé autour du travail, de la marchandise et de l'échange.

"...l'usage du mot " travail " pour désigner "gagnepain "se généralise à partir du XVIII siècle (Jacob, 1994), au moment même où la société traditionnelle se fait remplacer violemment par la société moderne." D'ailleurs, l'usage du mot " travail » pour désigner "gagne-pain » se généralise à partir du XVIII ${ }^{\mathrm{e}}$ siècle (Jacob, 1994), au moment même où la société traditionnelle se fait remplacer violemment par la société moderne. Dès lors, on ne travaille plus en tant que membre d'un groupe, chacun travaille maintenant pour sa propre bouchée de pain!

Le travail ne devient ainsi "travail » au sens moderne qu'avec le développement du marché et du capitalisme où l'individu, dépourvu des moyens de production, vend, pareillement à toute marchandise, sa force de travail au capitaliste. Dans sa conception occidentale contemporaine, le travail est propre à la modernité. Marx (1867) est probablement l'auteur qui en a développé la description la plus claire en théorisant au milieu du XIX ${ }^{\mathrm{e}}$ siècle la " force de travail " comme marchandise. Il développe ainsi 
quelque chose d'original à partir d'une critique épistémologique de Hegel et de Feuerbach. Pour Marx, la réalité est celle de la pratique, du faire, de l'acte, et non celle du discours, comme le concevait encore la pensée philosophique de l'époque. L'acte de l'individu est ainsi conçu comme étant le réel qui agit sur luimême. Or très concrètement, c'est ce qu'avaient compris dès le $\mathrm{XVIII}^{\mathrm{e}}$ siècle les marchands et les théoriciens du libéralisme à partir même des prémisses du libre arbitre de l'individu qui sont au centre de la société moderne :le réel s'incarne dans l'économie politique. Les économistes dirent en substance que la liberté se réalise dans l'action de chacun, plus précisément dans le travail de chacun. Autrement dit, l'économie politique du XVIII siècle affirme que la liberté réside tout entière dans le travail — dans la pratique sociale - impliquant, à la suite de Locke, le travail libre du propriétaire. Et Marx n'a eu qu'à formuler l'antithèse au libéralisme : "la liberté par le travail » lorsque le travail est "pour l'autre» (pour le propriétaire), n'est pas liberté, mais aliénation. D'où sa critique de l'économie politique qui expose les contradictions du travail "pour l'autre", du travail (ponos) de l'employé pour le compte de l'employeur.

On peut cerner trois moments décisifs qui poussent plus à fond l'organisation du travail comme aliénation : la révolution industrielle, la révolution managériale et la crise contemporaine ${ }^{8}$. Avant la révolution industrielle, le travail était centré sur le métier, tel que l'artisanat, la forge et l'agriculture. Ainsi, le résultat du travail de chacun demeure un produit concret et nécessaire à la vie de l'unité familiale.

\section{La division du travail et le travail aliéné}

"La bourgeoisie

envisage la production de façon rationnelle : produire massivement au moindre coût en vue de maximiser le profit."
La révolution industrielle consacre idéologiquement la domination du capitalisme, en associant le progrès technique à la production (Miguel et Ménard, 1988). La bourgeoisie envisage la production de façon rationnelle : produire massivement au moindre coût en vue de maximiser le profit. Pour cela, la classe dominante monopolise l'ensemble des ressources disponibles, dont 
la science et la technique, pour organiser le processus de travail, qui passe par l'innovation des techniques de production et par une division du travail toujours plus poussée. Le travailleur de métier se transforme en ouvrier d'usine : maintenant salarié, il travaille à la réalisation du "projet " de son employeur. Le travailleur se transforme ainsi progressivement en outil de la production. À partir de ce moment, le travail peut être compris comme abstrait, car il est détachable du travailleur (Pinard, 2000). La transformation du rapport humain à la nature est alors divisée en deux moments séparés de l'existence :la production et la consommation. De plus, la consommation aura moins tendance à satisfaire des besoins concrets qu'à exprimer une capacité abstraite de monnayer les moyens de consommer.

Les principaux développements organisationnels du travail de la phase industrielle du capitalisme sont ainsi typiquement des améliorations techniques. L'objectif est d'éliminer l'imprévu et donc d'organiser ou d'encadrer totalement le travail. L'initiative et l'autonomie individuelle sont réduites au maximum et le travail est complètement réfléchi par une autre personne pour maximiser son efficacité. Braverman (1976) a magistralement montré comment cela a été fait, secteur d'emploi par secteur d'emploi. Ce processus finira par s'étendre à tous les champs de l'activité humaine, y compris au travail intellectuel dans l'université (Readings, 1996).

Vient ensuite la révolution managériale avec son immense impact sur le fonctionnement de la société. Elle se rattache d'abord au secteur tertiaire avant d'être généralisée à l'ensemble de l'activité économique et de l'activité étatique. La révolution managériale naît aux États-Unis dans la foulée de l'implication

"Le capitalisme corporatif-managérial assume dès lors sa dimension politique : c'est l'ensemble de la société qui doit être organisé au service de l'économique." de l'État dans l'effort de guerre. Des normes plus sévères vont désormais s'appliquer aux entreprises ${ }^{9}$. De leur côté, les entreprises ne s'occupent plus seulement d'organiser la production, mais cherchent aussi à organiser le marché des produits ainsi que le marché du travail. Le capitalisme corporatif-managérial assume dès lors sa dimension politique : c'est l'ensemble de la société qui doit être organisé au service de l'économique. À ce titre, le modèle soviétique a poussé à la limite " la gestion totale " de la société vers des fins économiques (Grant, dans Pinard, 2000), qui 
conduit à une fusion entre les sphères privée et publique de la société. C'est ainsi que ce modèle de gestion s'est déployé jusqu'à la fin des années soixante-dix. (Pinard, 2000).

\section{La consommation et l'endettement comme aliénation supplémentaire}

Loin d'être maintenant en retrait, le modèle managérial prend aujourd'hui appui sur chacune des crises sociales et économiques pour approfondir son emprise sur nos vies. On assiste aujourd'hui à une financiarisation de l'ensemble de la vie sociale. Cela signifie que la norme de consommation ne se trouve plus dans le rapport fordiste (production de masse et consommation de masse) issu de la Deuxième Guerre mondiale. La norme prend désormais la forme de l'endettement qui se superpose à la surconsommation. Alors qu'à ses débuts les deux moments du rapport marchand prenaient la forme de la marchandise et de l'ouvrier médiatisé par l'argent (la consommation), la forme actuelle est celle d'une médiation par le crédit (l'endettement). Les managers des banques ont développé trois stratégies pour inclure les salariés dans le circuit financiarisé du capitalisme. Ils ont d'abord introduit la carte de crédit dans les années cinquante et, après que son usage se fut généralisé, il leur fallait ensuite élever le taux d'intérêt pour améliorer le taux de rendement du "produit crédit ", de $10 \%$ à $20 \%$. La carte de crédit a ensuite été libérée des banques particulières, devenant un service à part, autonome, si bien qu'il n'est plus nécessaire d'être client d'une banque pour avoir la carte de crédit. Finalement, les managers ont su accaparer et maintenir une clientèle spécifique d'" usagers qui ne remboursent pas l'entièreté de leur solde de compte. » (Pineault, 2012, p. 73) Ces derniers sont une immense source de profit pour les banques. Cela se comprend aisément, vu la différence entre l'intérêt payé par la banque à l'épargne (autour de $2 \%$ ) et l'intérêt perçu par elle pour le crédit (autour de $20 \%$ ).

La surproduction qui doit s'écouler dans la surconsommation a ainsi recours au surendettement généralisé des ménages salariés. "À la massification de la production et de la consommation 
"Cette nouvelle culture de la consommation à crédit passe également par une " déstigmatisation " de la faillite personnelle et une normalisation $d u$ surendettement. » s'ajoute dès lors celle du crédit comme nouvelle caractéristique du capitalisme avancé. » (Pineault, 2012, p. 75). Mais ce nouveau mode de régulation économique, " cette économie culturelle du crédit ${ }^{10}$ " (p. 76), donne un sens nouveau à la consommation, qui devient la soupape de l'aliénation au travail. De plus en plus d'individus trouvent ainsi leur liberté en consommant, trouvent une échappatoire à leur aliénation. Mais de plus en plus d'individus souffrent d'une telle pauvreté de la finalité instrumentale et matérielle de la vie contemporaine.

Cette nouvelle culture de la consommation à crédit passe également par une "déstigmatisation " de la faillite personnelle et une normalisation du surendettement. Rien ne traduit mieux cette évolution que l'émergence de l'expression populaire "maxed out", signifiant qu'un individu a atteint la limite de crédit d'une carte. Parfois cependant, «l'expression ne s'applique plus à l'objet, mais au sujet " (Pineault, 2012, p. 77). Dire "I'm maxed out " signifie que l'on est endetté à la limite et là, le stress peut être grand, plus encore si l'individu occupe un emploi précaire ou si l'atmosphère générale dans la société est plutôt inquiétante, comme lors d'une crise économique ou quand on invoque la menace économique de la Chine.

Ainsi la carte de crédit est le dispositif central de la «massification du crédit chez les salariés du XX $\mathrm{XX}^{\mathrm{e}}$ siècle » (Pineault, 2012). Un second dispositif de crédit est l'hypothèque, un troisième étant la ligne de crédit. L'objectif du système est de fournir le crédit sous toutes les formes possibles et imaginables, de manière à approfondir au maximum l'endettement des particuliers pour compter ensuite sur l'intérêt comme mécanisme d'accumulation. Dans ces conditions de la diversification généralisée des formes de crédits, c'est la culture humaine tout entière qui est en transformation. Pineault (2012, p. 80) remarque qu'ainsi, ce sont « les conditions subjectives d'une valorisation culturelle de l'instantanéité et de l'indétermination, typiques de ce que Zigmut Bauman a pu nommer les formes de la vie "liquide", qui caractérisent les sociétés capitalistes contemporaines avancées, surtout en Occident ». Tout ce système a également été au fondement de la crise financière de 2007 et toute la société s'est alors mobilisée pour remettre 
"Depuis trois siècles à peine, les rapports de l'Homme à son milieu, à sa communauté et aux valeurs ont été radicalement transformés. ” ce système financier sur ses pieds, en recourant aux impôts des contribuables pour recapitaliser le système.

Depuis trois siècles à peine, les rapports de l'Homme à son milieu, à sa communauté et aux valeurs ont été radicalement transformés. De l'artisan au manœuvre industriel salarié (XVIII ${ }^{e}$ et $\mathrm{XIX}^{\mathrm{e}}$ siècle), de ce dernier à l'ouvrier de production de masse (XIX ${ }^{\mathrm{e}}$ et $\mathrm{XX}^{\mathrm{e}}$ siècle) et au salarié précaire massivement endetté (XXI ${ }^{\mathrm{e}}$ siècle), nous avons assisté à la transformation d'une «identité qui perdure d'une classe de salarié non seulement exploité et aliéné, mais aujourd'hui également endetté, ces trois dynamiques se renforçant les unes les autres" (Pineault, 2012,p. 89). C'est dans cette aliénation généralisée aux deux pôles de la vie (production et consommation) que réside la souffrance psychique au travail. Travailler dans quel but?

Au Canada, plus d'un employé sur cinq déclare souffrir de dépression, largement à cause des pressions au travail (Ipsos Reid, octobre 2012), tandis que près des trois quarts (72\%) des Canadiens ressentent des niveaux de stress inconfortables au travail, chiffre qui monte à $90 \%$ pour les 18-24 ans. En particulier, les finances et la vie au travail sont identifiées comme étant responsables de cette situation (Ipsos Reid, novembre 2012). Le ratio dette-revenu est monté de plus de 55 points de pourcentage de 1990 à 2009. La croissance de l'endettement moyen des ménages s'est ensuite accélérée après 2002 : le ratio de la dette au revenu après impôt a atteint 148 \% en 2009 (Statcan, 2012), passant ensuite à $152 \%$ en 2010 et à 163,4\% en 2012 (Couture, 2012). Les familles monoparentales avec enfants avaient un ratio $227 \%$, comparativement à $170 \%$ pour les familles biparentales avec enfants (Statcan, 2012). Que faire de ces informations?

\section{Le discours contemporain et sa réalité}

Tout cela tranche avec le discours contemporain sur le travail. Il y a d'abord cette insistance sur la croissance économique, identifiée comme étant l'impératif absolu auquel nous devons sacrifier tous les efforts de la société. Ce point de vue est soutenu aussi bien 
"Il y a d'abord cette insistance sur la croissance économique, identifiée comme étant l'impératif absolu auquel nous devons sacrifier tous les efforts de la société."

"Le développement durable signifie aujourd'hui que c'est l'activité qui doit être continue (durable), au lieu de signifier que c'est le résultat de l'activité qui le serait." par le discours politique libéral dominant que par le discours social démocrate. Il faut augmenter la productivité, il faut réduire les dépenses de l'État, celles de l'entreprise, éliminer les déficits, réduire la dette de l'État, introduire les mesures d'austérité, chasser les fraudeurs, craindre la compétition des tigres de l'Asie, alors même que des pans entiers de l'économie occidentale sont délocalisés au Levant par "nos " gestionnaires de capitaux! (Rodin, 2011) Tous ont alors l'impression de vivre la précarité. Alors que nos parents et nos grands-parents avaient lutté contre les méfaits de la crise, alors qu'ils s'étaient sacrifiés à la guerre et qu'ils avaient négocié les conditions de vie et de travail décentes, alors que le contexte historique avait débouché sur la création d'un État social, voici que les plus récents développements de la société contemporaine créent plutôt la peur et la souffrance au travail.

Notre travail participe à la croissance économique continue, mais il nous épuise par son rythme. Aussitôt terminé le travail, nous courrons au loisir; aussitôt gagné notre salaire, nous courrons le dépenser. Ainsi "roule " l'économie de masse! Ainsi la mode s'empare de nous. Il nous faut voir la dernière production - et non plus création - culturelle, il nous faut posséder le dernier gadget électronique, la série des "i », les écrans plats, les lecteurs haute définition, le nouveau 3D, alors même que nous venons tout juste de nous habituer au VHS et au CD. Mais ceux-là sont obsolètes, déjà. Il y a les enfants à conduire à la piscine, au ballet, à la balle, à la musique; il y a les enfants qui veulent ce que le copain de classe a déjà obtenu.

Ainsi, tout doit être aligné sur la croissance économique, nos horaires de travail, notre statut, même l'économie verte doit se soumettre aux impératifs de la croissance économique, puisque ce type de développement, auquel on a emprunté le qualificatif de durable, dans le sens de continuité infinie, doit être soumis à l'instrumentalité du développement au lieu de l'être à son principe. Le développement est alors absolument réduit à la seule dimension quantitative, alors que l'écologie et son économie verte misaient sur sa dimension qualitative. Le développement durable signifie aujourd'hui que c'est l'activité qui doit être continue (durable), au lieu de signifier que c'est le résultat de l'activité qui le serait. 
On ne fait pas des choses solides et bien faites qui vont durer, mais des choses jetables que nous devrons continuer à produire.

\section{Un remède à la souffrance au travail : une conception humaine de la vie en société}

"Le développement devrait toujours être compris comme celui des trois éléments inhérents à la vie des sociétés : culturel, politique et économique.»
Reprenons donc une autre fois le sens de notre critique globale à l'ensemble (totalité) de la société contemporaine, ainsi que le rôle que nous voulons dégager pour le service social. D'abord, nous voulons réaffirmer que le développement actuel du monde pose problème. Le développement auquel nous assistons est inégal, seuls quelques secteurs de chaque société, seuls quelques secteurs du monde et seule une minorité (1\%?) en profite au plein sens du terme. Ensuite, ce développement ne respecte pas les fondements naturels de la vie :il est polluant et menace la survie de nombreuses espèces vivantes, tant la vie animale et végétale que la vie humaine (Cafaro et Crist, 2012;Zapf, 2009). Finalement, le développement auquel nous assistons est partiel; il est à peu près strictement compris dans sa seule dimension économique, alors que le développement est aussi culturel et politique. «Culturel » doit ici être compris non pas dans son sens esthétique, mais dans son sens anthropologique impliquant une forme particulière d'ontologie qui distingue par exemple les sociétés archaïque, traditionnelle et moderne. Le développement devrait toujours être compris comme celui des trois éléments inhérents à la vie des sociétés : culturel, politique et économique. Il faut donc comprendre ici ces trois concepts dans cet ordre précis, le premier référant à l'ontologie (le rapport au monde de l'individu), le second à la normativité (que l'on pourrait qualifier de lien à la transcendance, qui permet la création de sens collectif) et le troisième à l'instrumentalité dans la reproduction de la vie. Or, depuis deux siècles, nous avons renversé cet ordre : nous partons aujourd'hui de l'instrumentalité du rapport au monde, qui est l'opérationnalisation du libre arbitre dans la lutte pour la satisfaction des besoins matériels. C'est ainsi que nous sommes, efficacement, instruments du travail pour l'autre, aliénés et souffrants au travail et dans la vie. C'est ainsi que la 
transcendance tend à complètement disparaitre et que l'ontologie se replie sur elle-même. Le développement fondé sur l'intérêt de l'entrepreneur individuel est pour chacun de nous non-sens.

La question de la qualité de la vie apparait également comme nécessitant la présence d'un État de droit puissant, fondé sur la volonté interventionniste vers un commonwealth - au sens où l'entendait Hobbes, d'un bien-être civilisationnel collectif capable de valoriser le développement pour tous, de circonscrire l'action des intérêts privés, des corporations, des entités particulières, au nom du droit à la qualité de vie de l'ensemble de la population de la Terre ${ }^{11}$. La question de la qualité de la vie est ainsi celle de la sociabilité, de la capacité des petites entités de prendre des décisions pour elles-mêmes dans le cadre d'une réappropriation des déterminantes de leurs vies. Il faut voir les grandes mégalopoles du monde comme Paris, New York, Shanghai, Tokyo, Jakarta, Rio de Janeiro, Mexico, Mumbai, Dacca, Shenyang, Bangalore ou Delhi ${ }^{12}$, pour comprendre comment le développement peut parfaitement conduire à une dégradation des conditions de vie.

La déstructuration de l'État et de la socialité par le néolibéralisme qui depuis les années 1980 insiste sur un large retrait de l'État pour laisser la place à l'entreprise privée va à l'encontre d'une conception humaine de la vie en société. La nature humaine n'est pas la capacité de se vendre sur le marché du travail, elle ne se réduit pas à un pouvoir d'achat, mais elle est constitutive d'une subjectivité, fondées sur des valeurs, d'une capacité à prendre des

"La domination de l'ensemble de la société et de son développement par les marchés est une invention récente et dangereuse; elle n'est que celle d'une partie de la population..." décisions collectives sur l'orientation de la société, en partant de la nécessité de répondre aux besoins réels et complets des populations, dans le contexte des limites qu'impose la nature. La domination de l'ensemble de la société et de son développement par les marchés est une invention récente et dangereuse; elle n'est que celle d'une partie de la population, d'une classe sociale, la bourgeoisie ou, pourrions-nous dire pour être plus précis en ce début de $\mathrm{XXI}^{\mathrm{e}}$ siècle, la classe des super riches (1\%?) que l'on peut qualifier d' "over class", tellement elle vit une réalité abstraite au regard de la condition humaine générale. 
"...nous sommes

le produit de notre existence historique et collective, bénéficiaires de ceux qui nous ont précédés et testateurs de ceux qui nous suivront. »
Ce qu'oublient en fait les apôtres du marché lorsqu'ils se rendent au village global en passant par l'autoroute électronique pour y effectuer des transactions financières, c'est que la présence de l'Homme sur la Terre n'est ni gratuite ni exempte de sens. En fait, c'est là que la définition de l'Homme puise toute sa force et son mystère. Tous les Hommes avant la naissance du banquier, du premier qui a pris conscience de la force de l'orage et de la chaleur du feu à ceux qui ont écrit les "Droits de l'Homme ", savent qu'il y a quelque chose de mystérieux dans la vie. L'animisme a nommé ce mystère " esprit ", la religion l'a nommé " dieu ", d'autres encore se limitent à le nommer «nature » ou «vie » et respectent ce mystère comme s'il n'y avait rien de plus précieux. Marcel Gauchet (1977) a nommé cette disposition générale « la dette de sens ». Par là, cet auteur voulait simplement dire que l'individu, tel qu'il existe socialement, doit cette existence à quelque chose de plus grand que lui, à quelque chose de plus important. L'animisme et la religion conçoivent déjà que nous devons notre existence à une "volonté » qui nous est extérieure : grâce aux ancêtres ou à Dieu, notre existence individuelle nous a toujours échappé. Nous savons aujourd'hui que nous sommes largement la médiation par un corps et un esprit de quelque chose d'accumulé et de transmis qui par nature n'est pas autonome. C'est cela la "dette de sens", c'est-à-dire que l'individu est dans la société beaucoup plus qu' "individu ", il est porteur d'une totalité héritée, responsable d'elle devant ses propres héritiers. Le libre arbitre, s'il se déploie à l'extérieur d'un cadre normatif collectif et historique, ne peut gérer cet héritage, il ne peut que le conduire à sa perte, par oubli et par manque d'intérêt (Freitag, 2011).

L'implication de la critique de Freitag et de la formule de Gauchet est simple : elle signifie un rejet des prémisses de l'individualisme de la doctrine libérale. L'individu doit son existence à son appartenance à une multitude et à une entité qui le dépasse. Nous ne sommes pas les maitres absolus de qui nous sommes, nous sommes le produit de notre existence historique et collective, bénéficiaires de ceux qui nous ont précédés et testateurs de ceux qui nous suivront. Tous ne sont pas égaux, certains sont plus talentueux, certains sont même chanceux. 
Mais les classiques et les grandes œuvres, la capacité à résoudre les énigmes de l'univers, celle d'explorer le cerveau et celle de construire les monuments de notre époque, tout cela relève moins des accomplissements individuels que des réalisations cumulatives de l'Homme. Chaque individu n'est donc pas seul, à partir de son libre arbitre, responsable de ses choix devant la totalité à laquelle il répond.

L'espèce humaine semble être à une croisée des chemins. Nous devons prendre des décisions qui assureront notre développement sain à long terme. Il nous faut faire cela en ayant en tête la totalité du développement, celui de toute l'humanité et de toute la nature, telle que cette totalité se synthétise ici et maintenant en chacun de nous. Il faut que l'Homme parvienne à faire cela en toute humilité, en partant du point de vue que nous avons une "dette de sens " envers toutes les formes de la vie, toutes celles qui existent, toutes celles qui ont existé, y compris toutes celles que l'Homme a symboliquement inventées pour créer le sens de sa vie. Nous avons aussi une dette envers ceux qui nous remplaceront, nous devons leur léguer une société saine, sur une planète saine et contenant toujours un avenir indéterminé. Nous devons resituer la conception du bonheur dans la perspective des rapports aux autres Hommes, nous devons resituer le sens de la vie dans nos rapports à l'ensemble de la vie et recomposer notre existence dans la trame de notre développement anthropologique. Le bonheur ne réside ni dans les réalisations matérielles ni dans l'accumulation sans fin des richesses. D'ailleurs, pourquoi insister, le dicton le dit depuis longtemps : même s'il aide, l'argent ne fait "Le service social a ainsi un rôle normatif tout tracé dans ce grandiose plan de transformation de l'humanité et de son milieu : participer à réencastrer la solidarité dans l'ensemble de la structure sociale. » pas le bonheur!

Le service social a ainsi un rôle normatif tout tracé dans ce grandiose plan de transformation de l'humanité et de son milieu : participer à réencastrer la solidarité dans l'ensemble de la structure sociale. C'est par ces petits bouts de contributions qui consistent à dénoncer l'individualisme et à favoriser des solutions collectives; à dénoncer la pauvreté et à favoriser des mesures de redistribution des richesses; à dénoncer toutes les formes de violence et à favoriser le développement de compromis et de solution alternative; à dénoncer la guerre, l'analphabétisme, 
le désœuvrement, le gaspillage, la corruption, le népotisme et à favoriser le développement des qualités humaines; à ne plus tolérer que certains s'approprient les moyens du développement de l'ensemble pour leurs propres bénéfices; à ne plus tolérer le développement matériel en soi au profit de l'autre. C'est en faisant cela que chacun peut contribuer à changer le monde.

«Il est contre la Loi de la nature qu'un enfant commande un vieillard, qu'un imbécile conduise un Homme sage et qu'une poignée de gens regorge de superfluités tandis que la multitude affamée manque du nécessaire ", écrivait Rousseau en 1755 (1964, p. 194). Le rôle contemporain des travailleurs sociaux est de participer à ce que cessent les aberrations individualistes qui caractérisent la société actuelle et à ce que se développe la conscience sociale d'être membre d'un tout. Tout cela, et tant encore, fait partie de la contribution de la profession au développement du bien-être et du mieux être de la société. La solidarité n'est pas une option, elle ne l'a jamais été. Contre la domination du libre arbitre absolu émancipé des contraintes sociales et historiques, la solidarité est la manière d'atteindre notre plein potentiel humain : elle est la manière humaine de vivre dans le monde. Elle est ainsi la cure contre la souffrance au travail.

\section{Notes}

1 Nous n'aborderons pas la question de la conscience que plusieurs philosophes rattachent à ces concepts. Nous dirons simplement que l'en-soi et le pour-soi sont des concepts qui désignent respectivement ce qui ne possède pas de liberté et ce qui en possède. Étudier une chose en soi consiste à l'étudier comme si elle était donnée une fois pour toutes, telle quelle. Ainsi, le travail conçu en soi serait une activité nécessaire à la vie comportant un très grand niveau de contrainte. Étudier une chose pour-soi consiste à l'étudier en comprenant sa dimension libre, sa capacité à devenir autre ou à être transformée. De cette manière, le travail conçu pour-soi serait une activité auxiliaire à la vie comportant un très grand niveau de liberté.

2 Homme, comme dans «Droits de l'Homme », avec la majuscule, désigne le générique de l'espèce et non le sexe masculin.

3 Nous ne posons pas la question en termes absolus, il ne s'agit pas de choisir l'un des termes (la valeur ou la finalité) et de rejeter l'autre. Il s'agit plutôt de penser leur rapport : est-ce que la finalité est dépendante ou indépendante des valeurs dans une société donnée? Par exemple, l'Homme a très longtemps utilisé les gains de productivité pour augmenter son temps de repos (spirituel), nous utilisons ces gains pour augmenter la richesse (matériel). Il y à là deux rapports différents entre valeur et finalité du travail. 
4 L'ontologie se définit le mieux ou le plus clairement comme étant « la logique du maintien de soi dans l'existence " (Fillion, 2006, p. 69). Ajoutons que la méthodologie ici choisie n'est pas celle de l'individualisme méthodologique de Weber, elle ne s'inspire que de son antipositivisme.

5 Le mythe ne comporte généralement pas de "fin ", comme dans "la fin du monde ». Dans le mythe, il n'y a qu'une finalité de retour sur soi :le mythe, narration de la totalite de la communauté, sert à rappeler, à corriger ou à affirmer l'ordre de cette communauté.

6 Ils sont nombreux «les chanceux » qui aujourd'hui peuvent se permettre des vacances de chasse ou de pêche pour «sortir du travail ». La pêche et la chasse sont largement considérées comme des activités ritualisées, comme en fait foi l'exclusion des femmes de ces activités.

7 Cela est illustré à la perfection dans la Réforme, où l'individu a maintenant accès direct à Dieu, sans la médiation de l'institution.

8 Sur ces points, nous suivons les arguments de Pinard (2000) et de Pineault (2012).

9 Nous pouvons ajouter ici que le pendant de l'implication des États à l'entreprise a pour corolaire le fordisme, c'est-à-dire l'adoption d'une norme sociale de consommation, d'une norme sociale d'imposition des entreprises, ainsi que la reconnaissance généralisée du syndicalisme. Tout cela se concrétise sous la forme de l'État keynésien, aussi dit État social ou État providence. L'essentiel demeure le compromis social issu de la transformation du rapport de force à partir du moment où la crise du libre marché s'accompagne d'une crise sociale et d'une guerre majeure et où l'État doit tout reprendre en main. C'est ce nouvel équilibre qui a sera à nouveau transformé à la fin des années soixante-dix.

10 Paradoxe contemporain, les individus peuvent s'endetter jusqu'au cou, mais les États doivent rembourser leurs dettes, réduire leur déficit " au plus vite ".

11 Ici, l'espace manque pour indiquer qu'une qualité de vie plus grande, une fois l'intérêt privé circonscrit, passe ensuite par une importante réduction de la présence de l'État pour laisser libre cours au développement des autoservices collectifs et locaux.

12 Cette liste est une liste partielle des villes du monde qui comptent plus de 10 millions d'habitants en 2000. Il est estimé que le nombre de mégalopoles triplera d'ici un demi-siècle et que la majorité de ces villes seront en Asie. Comment alors, de manière réaliste, réfléchir la démocratie, comment faire face à la demande alimentaire sans avoir recours aux OGM, aux engrais chimiques et à la nourriture industrielle? Comment penser que ces milliards d'individus ne voudront pas, eux aussi, vivre la civilisation de l'énergie fossile.

\section{Bibliographie}

BRAVERMAN, Harry (1976). Travail et capitalisme monopoliste : la dégradation du travail au XXe siècle, Paris, Maspero, $360 \mathrm{p}$.

CLASTRES, Pierre (1974). La société contre l'État, Paris, Les Éditions de Minuit, 190 p.

CAFARO, Philip, et Eileen CRIST (2012). Environmentalists confront overpopulation, Athens and London, The University of Georgia Press, $352 \mathrm{p}$.

CAILLÉ, Alain (1986). Splendeur et misère des sciences sociales : Esquisses d'une mythologie, Paris, Droz, $411 \mathrm{p}$. 
CAILLÉ,Alain, et Philippe CHANIAL (dirs.) (2009). L'Université en crise : mort ou résurrection? Revue du M.A.U.S.S, $N^{\circ}$ 33, Paris, 384 p.

COUTURE, Pierre (2012). "Le taux d'endettement des ménages atteint un record ", Le Soleil, 16 octobre 2012, réf. du 21 février 2013, http://www.lapresse.ca/le-soleil/affaires/ consommation/201210/15/01-4583623-le-taux-dendettement-des-menages-atteint-un-record. php.

ÉLIADE, Mircea (1963). Aspect du mythe, Paris, Gallimard, 246 p.

FILLION, François (2006). Sociologie dialectique : introduction à l'auvre de Michel Freitag, Montréal, Éditions Nota Bene, 333 p.

FREITAG, Michel (2011). L'abime de la liberté, Montréal, Liber, 510 p.

FREITAG, Michel (2007). "Combien de temps le développement peut-il encore durer? ", Société, No 27, Automne 2007, Montréal, Éditions Nota Bene, p. 211-241.

FREITAG, Michel (1995). Le naufrage de l'université et autres essais d'épistémologie politique, Montréal, Nota Bene, 368 p.

FREITAG, Michel (1989). "La nature de la technique et le problème normatif posé par son émancipation contemporaine dans le technologisme et le technocratisme ", Société, Hiver 1989, No 4, Québec, Groupe autonome d'édition, p. 7-94.

FREITAG, Michel (1987). "Les sciences sociales contemporaines et la question de la normativité ", Sociologie et sociétés, Vol. XIX, № 2, octobre 1987, Montréal, Presses de l’Université de Montréal, p. 15-35.

GAGNÉ, Gilles (1999). Main basse sur l'éducation, Montréal, Nota Bene, 111 p.

GAUCHET, Marcel (1977). «La dette de sens et les racines de l'État », Libre, No 2, Paris, Petite Bibliothèque Payot, p. 5-43.

GODBOUT, Jacques, et Alain CAILLÉ (1992). L'esprit du don, Paris, Éditions La découverte, 344 p.

GORZ,André (1988). Métamorphoses du travail — Quête du sens : Critique de la raison économique, Paris, Galilée, 302 p.

GOULDNER, Alvin W. (1970). The coming crisis of Western sociology, New York, Basic Books, 528 p. HALLPIKE, Christopher R. (1979). Foundation of primitive thought, Oxford, Clarendon Press, 516 p.

HARRISON, Jane Ellen (1991). Prolegomena to the study of Greek religion, Princeton, Princeton University Press, $682 \mathrm{p}$.

IPSOS REID 30 octobre 2012 Plus d'un employé canadien sur cinq déclare souffrir de dépression, réf. du 21 février 2013, http://www.newswire.ca/en/story/1048799/plus-d-un-employe-canadiensur-cinq-declare-souffrir-de-depression-les-gestionnaires-interviennent-davantage-mais-il-fautencore-plus-de-soutien-selon Ipsos Reid

IPSOS REID 5 novembre 2012. Three-quarters (72\%) of Canadians are experiencing an uncomfortable level of stress; Number jumps to nine in ten (90\%) among 18-24 year olds, réf. du 21 janvier 2013, http://www.ipsos-na.com/news-polls/pressrelease.aspx?id=5870

JACOB, Annie (1994). Le travail, reflet des cultures : Du sauvage indolent au travailleur productif, Paris, Presses Universitaires de France, 276 p.

LOCKE, John [1690] (1999). Traité du gouvernement civil, traduction de David Mazel, Paris, GarnierFlammarion, $381 \mathrm{p}$.

MARX, Karl [1867] (1976). Le capital, traduction de Joseph Roy, Paris, Éditions Sociales, 317 p. 
MAUSS, Marcel (1960). Sociologie et anthropologie, Paris, Presses Universitaires de France, 552 p. MIGUEL, Christian, et Guy MÉNARD (1988). Les ruses de la technique, Montréal, Boréal, 388 p. PINARD, Rolande (2000). La révolution du travail : De l'artisan au manager, Montréal, Liber, 323 p. PINEAULT, Éric (2012). "Financiarisation, crédit et théorie critique du capitalisme avancé ", dans Francis Dupuis-Déri (dir.), Par dessus le marché! Réflexions critiques sur le capitalisme, Montréal, Écosociété, p. 49-89.

READINGS, Bill (1996). The university in ruins, Cambridge, London, Harvard University Press, 256 p. RODIN,Étienne (2011). L'horreur managériale : gérer, instrumentaliser, détruire, Édition l'Échappée, 128 p. ROSANVALLON, Pierre (1981). La crise de l'État-providence, Paris, Seuil, 183 p.

ROUSSEAU, Jean-Jacques [1755] (1964). «Discours sur l'origine et les fondements de l'inégalité parmi les Hommes ", Euvres Complètes, Tome III, Paris, Gallimard.

SAHLINS, Marshall (1976). Âge de pierre, âge d'abondance : L'économie dans les sociétés primitives, Paris, Gallimard, $420 \mathrm{p}$.

STATCAN (2012). Tendances sociales canadiennes : Endettement et types de familles au Canada, réf. du 21 février 2013, http://www.statcan.gc.ca/pub/11-008-x/2011001/article/11430-fra.htm

TRIGGER, Bruce (1991). Les enfants d'Aataentsic : l'histoire du peuple Huron, Montréal, Libre Expression, $971 \mathrm{p}$.

VERNANT, Jean-Pierre (1965). Mythe et pensée chez les Grecs : Étude de psychologie historique, Paris, Maspéro, $428 \mathrm{p}$.

WEBER, Max (1917) (1959). Le savant et le politique, Paris, Plon, 224 p.

WEBER, Max (1904) (1965). Essais sur la théorie de la science, Paris, Plon, 539 p.

ZAPF, Michael Kim (2009). Social work and the environment: Understanding people and place, Toronto, Canadian Scholars' Press, 300 p. 